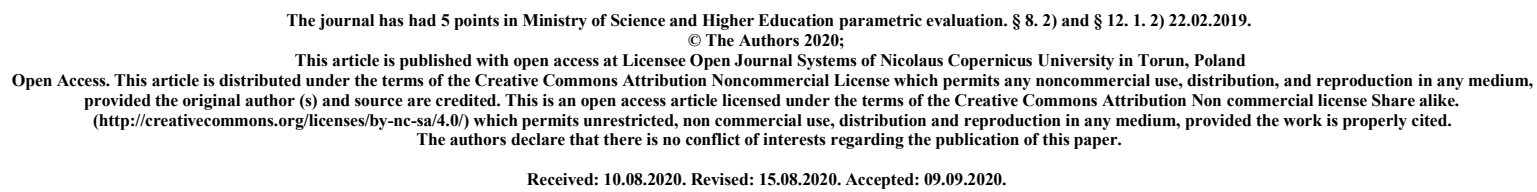

\title{
The number of passively smoked cigarettes and the risk of lung cancer among the inhabitants of the Lubelskie Region (2013-2017)
}

Robert Chudzik

Affiliation Chair and Department of Thoracic Surgery, Medical University, Lublin

Country Poland

Bio Statement -

Principal contact for editorial correspondence.

Beata Rybojad

Affiliation Department of Anaesthesiology and Intensive Care, Children's University Hospital, Lublin

Country Poland

Bio Statement -

Paweł Rybojad

Affiliation Chair and Department of Thoracic Surgery, Medical University, Lublin, Poland

Department of Thoracic Surgery, Holy Cross Cancer Centre, Kielce

Country Poland

Bio Statement - 
According to WHO, clean air is considered as one of the basic criterion influencing human health. Airborne particulate matters (PM) 2,5 are mixtures of organic and inorganic particles smaller than $2.5 \mu \mathrm{m}$. They have broad negative effect on health especially on respiratory and cardiovascular system. They could increase the sensitivity to air ways infections, worsen: allergic diseases, hypertension, myocardial infarction and also increase the risk of neoplastic diseases. NO2 pollution arises mainly during burning of fossil fuels, which is mainly related to road transport. On the basis of numerous scientific research, it is believed that the long term exposure to NO2 could increase inflammation and eventually lead to higher cancer morbidity.

We analyzed the data available in the Polish National Cancer Registry (PNCR), Chief Inspectorate for Environmental Protection and Air Quality Guidelines, We checked air pollution by the means of PM2.5and NO2 and thanks to mathematical equation delivered by Saskia C. van der Zee converted them into number of passively smoked cigarettes. Above data we compared to lung cancer morbidity in Lubelskie Region.

Based on the latest available data and literature, we can conclude that in 2013-2017 each inhabitant of Lubelskie Region smoked average 8 cigarettes a day $+/-2$. The incidence of lung cancer is more or less the same number. After 75 years everyone in the exanimated region had 30 package-years of passive smoking and high risk of lung cancer. Over same period of time cumulative risk of lung cancer according to PNCR were in women almost $2 \%$ and in men almost $6 \%$

Air quality in Poland is not satisfactory, exceeding the standards presented in the WHO Guidelines 2005, we can assume that this translates into a high risk of the incidence of lung cancer among our inhabitants.

Key words: lung cancer; air pollution

\section{INTRODUCTION}

Air cleanliness is recognized as one of the important parameters of health and welfare criteria. The World Health Organization has been dealing with the topic of air quality since 1987, when the first guidelines on standards and norms for pollution were issued. Since then, many scientific studies on the harmfulness of numerous substances which are largely the result of our activities, especially the development of technology and industry have been created. Since the first guidelines, the public has realized how important it is to care for the quality of the surrounding environment. This issue is gaining importance all the time. [1]

Nitrogen oxides mainly represented by nitrogen dioxide are pollutants mainly attributed to road transport. The effects of exposure to elevated concentrations are manifested in all age 
groups and even may appear in utero as low birth weight, low weight in relation to gestational age or premature delivery. It also increases the risk of children hospitalization due to exacerbations of respiratory diseases. In adulthood, due to long-term exposure to car exhaust fumes, risk of cardiovascular disorders. We should not forget about the increased risk of cancer, including lung and breast cancers. [1,2,3,]

Particulate matters are mixtures of organic and inorganic substances, including toxic ones such as dioxins, furans or numerous heavy metals. There are two most common types: PM 10 - particles up to 10 micrometers in diameter - can reach the upper respiratory tract and lungs. And PM 2.5 are particles with a diameter of up to 2.5 micrometers. Due to their small size they can get not only into the lungs, but also into the circulatory system. The main sources of dust pollutants is coal burning, particularly of low quality in old poorly regulated boilers, as well as the chemical, energy and mining industries. The numerous consequences of exposure to these aerosol matters include: circulatory arrhythmias, ischemic stroke, respiratory system disorders: sore throat, cough, exacerbation of COPD, lung cancer. According to the guidelines proposed by the World Health Organization, the annual average concentration for PM 2.5 should not exceed $10 \mu \mathrm{g} / \mathrm{m} 3$. [1,4,5,6]

Due to the nature and authority of the organization, when writing about air quality, we refer in many places to the standards of the World Health Organization issued in 2005. Other standard proposals that we could take into account were the guidelines adopted by the European Commission under Directive 2008/50 / EC of 2008 and the Regulation of the Ministry of the Environment of Poland of 2012 (Journal of Laws of 2012, item 1032). Unfortunately, these values are more liberal than those proposed by the WHO. Presumably, the explanation for this fact may be the economic nature of the European Union and the desire to care for the Polish economy which is based on coal as the main source of energy. Unfortunately, caring for the environment comes with costs and limitations. When reading the values of permissible exceedances of the standards in the EU and Poland and WHO, it is worth mentioning that only WHO defines each exceedance as harmful to human health.

\section{MATERIALS AND METHOD}

We started our work by query of literature on air pollution and its relationship to neoplasms, especially to lung cancer. Then we analyzed the guidelines for air pollutants presented by WHO in 2006 [7], we compared them to the Polish Environmental Protection Law published April 27, 2001. [8] Measurement values of air pollutants (PM 2.5; NO2) for Poland in 2013-2017 were taken from the database of the Chief Inspectorate for Environmental Protection. [9] Presented values of PM2.5 are the average of $24 \mathrm{~h}$ survey gathered from all measurement stations in Lubelskie Region. The completeness of the measurements is over $90 \%$. In the case of NO2, the annual average values based on survey performed every 1 hour from all measurement points in Lubelskie Region, The completeness of measurements in our survey was over $90 \%$, except for 2015 when the completeness was $75 \%$. The number of cases and the cumulative risk for lung cancer (ICD10 - C34) were reported by the National Cancer Registry (2013-2017). [10] Statistical studies were carried out using a Statistica.

Based on the available data, using the formula prepared by Saskia C. van der Zee, Paul H. Fischer, Gerard Hoek in the work "Air pollution in perspective: Health risks of air pollution expressed in equivalent numbers of passively smoked cigarettes" [11], we converted average concentrations of PM10 and PM 2,5 into number of passively smoked cigarettes by residents of Lubelskie Region during the period of one year. 
The latest available data from the Chief Inspectorate for Environmental Protection come from 2018, however, due to the fact that in the case of the National Cancer Registry, these are data from 2017, this study does not include 2018.

\section{RESULTS}

The etiology of lung cancer is multifactorial. We focused on air pollution and its influence on lung neoplasms. We took into account those substances that allow us to calculate the number of passively smoked cigarettes (NO2, PM2,5). The formula which was used in this surey also takes into account the the Black Carbon concentration, but Polish measuring stations do not perform this measurement. WHO has published guidelines for level of pollutants in the air, including the following: PM 2.5; NO2. [7] It is worth to notice that the European Commission also published its proposals for standards, which, however, are more liberal than those proposed by WHO. The standards for Poland provided by the 2001 Environmental Protection Law are similar to the European ones. [12] Standarts proposed by European Commission and individual countries are often corrected by economic issues while WHO focuses its activities on health problems and that is why its norms are more restrictive.

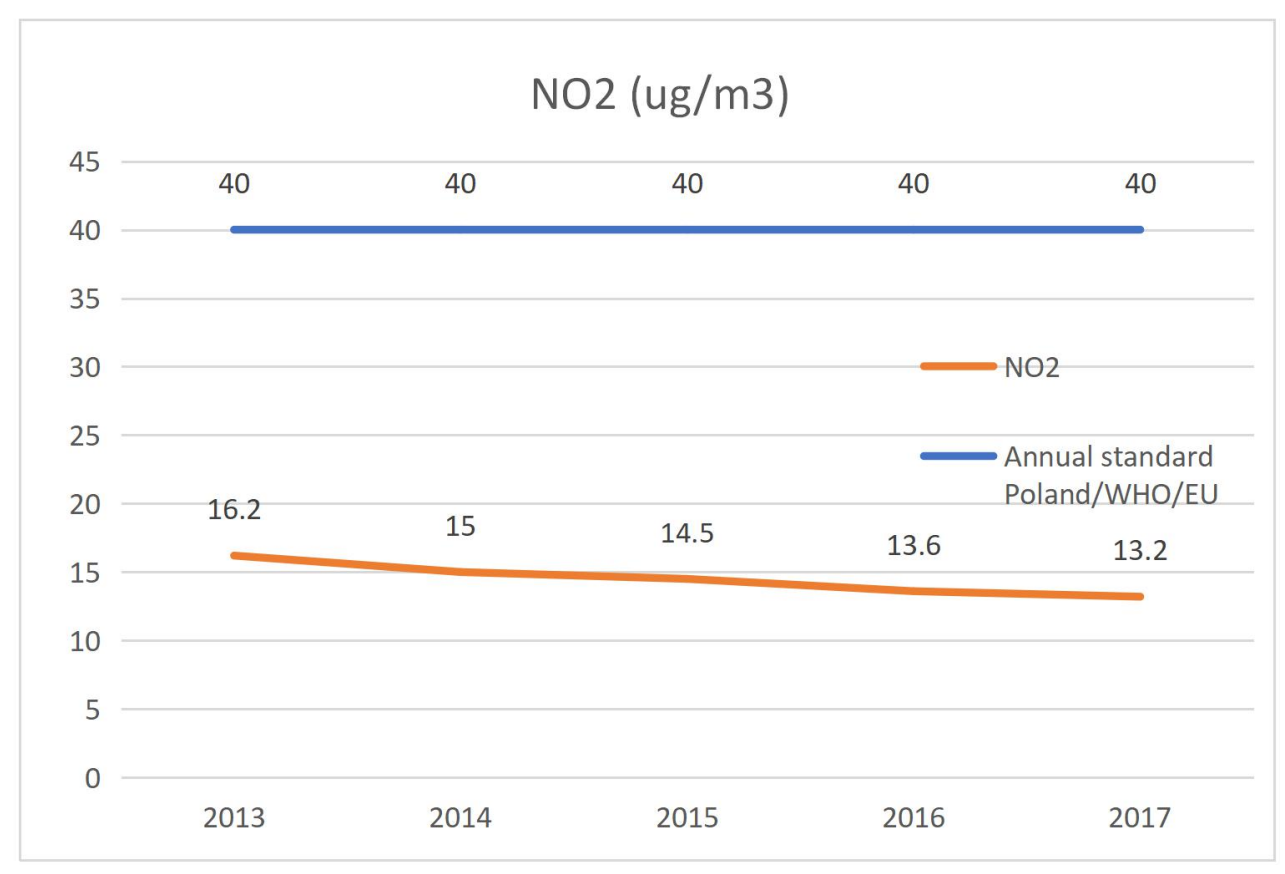

Figure 1. Average annual concentration of NO2

We can see a slowly but systematic decrease in the annual average NO2 concentration, but it should be noted that the data from 2015 is only 75\% completed. Guidelines of normal concentration of this pollutant provided by WHO, Poland and Europe have been met over the years. 


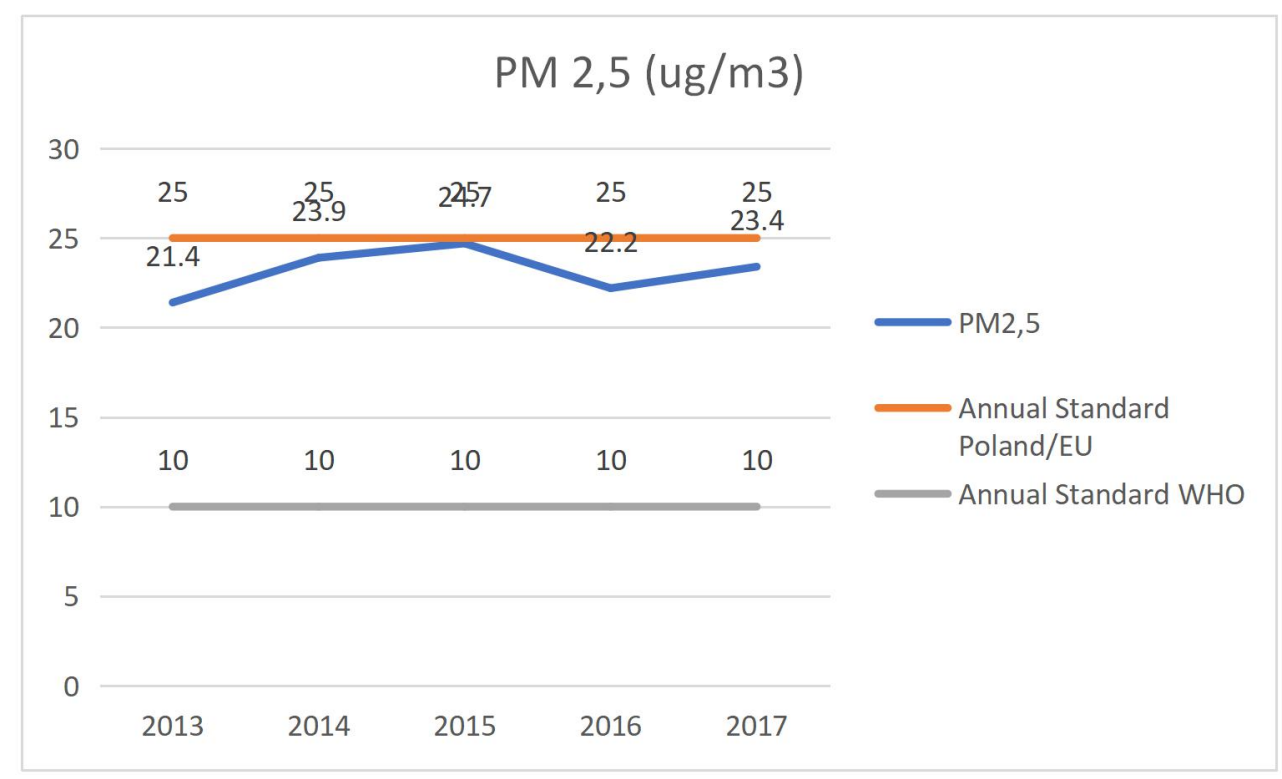

Figure 2. Average annual concentration of PM 2,5

In the case of PM 2.5, the annual average concentration remains at a similar level. There is a significant difference in the guidelines according to WHO, Polish and EU. These more liberal standards were met, but the more restrictive ones, proposed by WHO unfortunately were not.

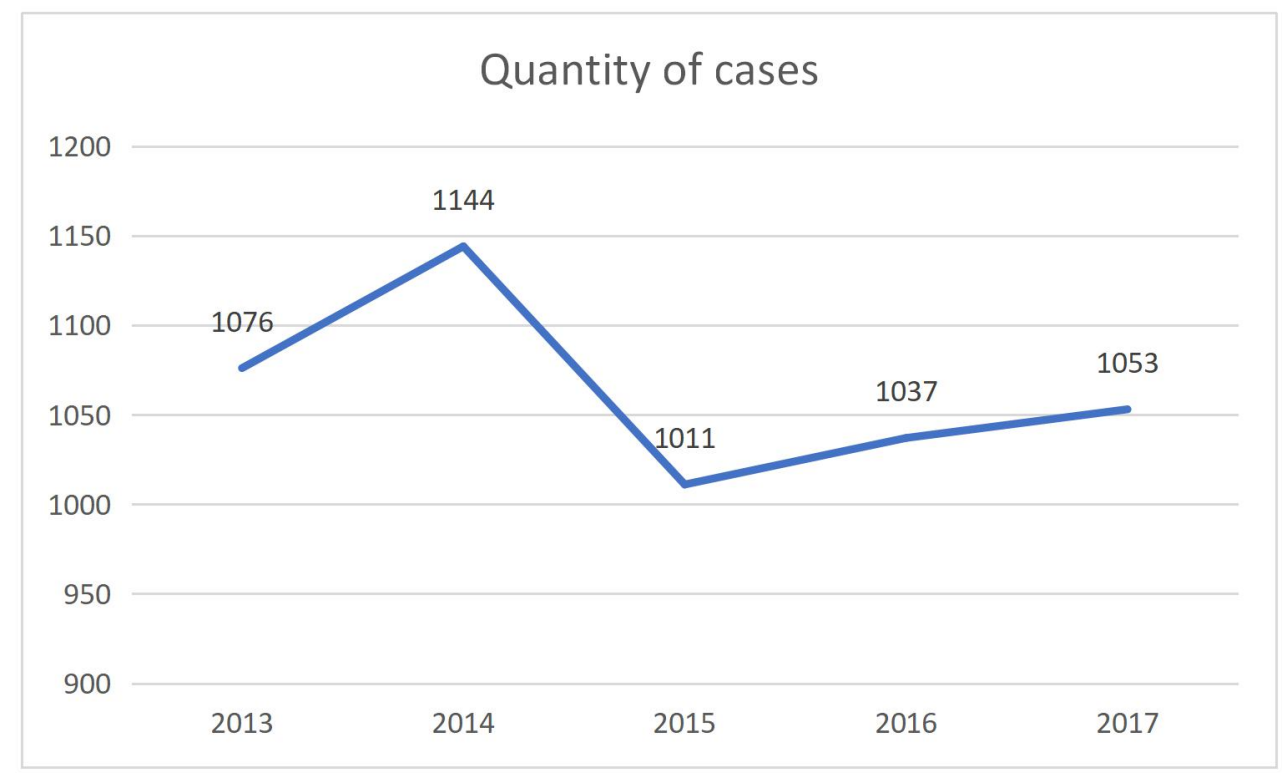

Figure 3. Quantity of cases C33 


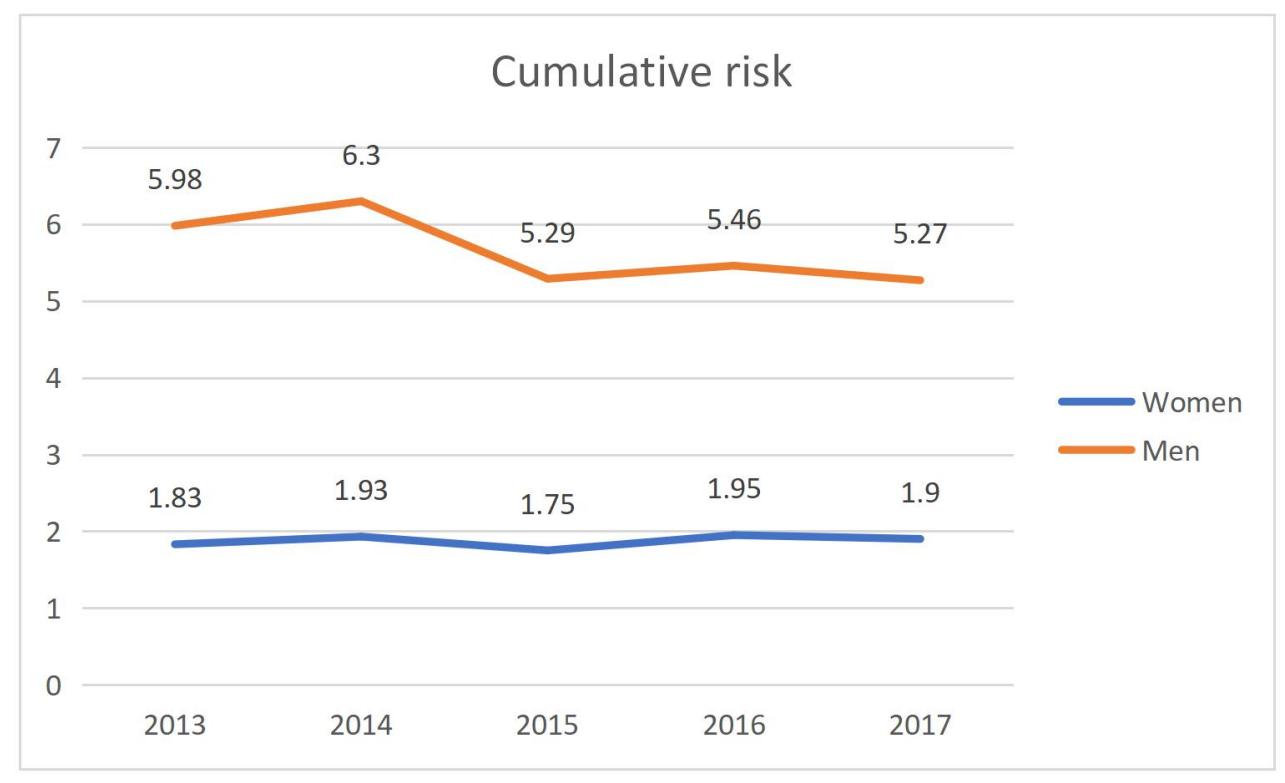

Figure 4. Cumulative risk for lung cancer

The average number of lung cancer patients in the presenting period was 1,064 cases each year. As we can see from the cumulative risk, men are responsible for the majority of recorded cases. However, over the years, the increase in the number of women in relation to the baseline data is alarming.

Table 1. Calculated amount of 'passively' smoked cigarettes

\begin{tabular}{|l|l|l|l|l|l|}
\hline & 2013 & 2014 & 2015 & 2016 & 2017 \\
\hline $\begin{array}{l}\text { Calculated } \\
\text { amount of } \\
\text { 'passively' } \\
\text { smoked } \\
\text { cigarette }\end{array}$ & 7.9 & 8.5 & 8.6 & 7.8 & 8.1 \\
\hline$+/-$ & 1.8 & 2 & 2.1 & 1.9 & 2 \\
\hline
\end{tabular}

In the analyzed years, based on the formula proposed by Saskia C. van der Zee et al. we calculated that air pollution with PM 2.5 and NO2 is responsible for passive smoking of approximately 0.4 packs of cigarettes a day. Therefore, after the age of 75 , every inhabitant of the Lubelskie Region has unconsciously smoked out 30 pack-years, which is a significant factor increasing the risk of lung cancer incidence.

\section{DISCUSSION:}

The state of air purity in the Lubelskie Region and in the whole Poland requires further thorough attention. Our study shows that in the case of NO2, the concentration trends are slowly decreasing, PM2.5 unfortunately are not which should be a cause for concern. Unfortunately we cannot assess in which part value of the measurements is caused by the 
pollution produced in Lubelskie Region itself and which part is caused by flow of air masses from the neighboring areas. Due to the agricultural nature of the Lubelskie Region and minor amount of heavy industry, we can assume that the main sources of pollution come from outside the studied area. On the other hand this is agricultural region with rather poor population. This also generates pollution by improper burning of coal and use of agricultural machines using majorly diesel fuel.

Similarly, we cannot clearly determine whether the increase in lung cancer incidence is caused by exposure to harmful substances in the Lubelskie Region itself or during migration to other parts of Poland or different countries. The intense migration we are currently dealing with also increases the level of NO2 (road transport). We should carefully follow the information on the air condition in the context of various standards, and also examine what exactly the differences between the individual norms result from. In this study, we drew attention to the persistent problem of lung cancer incidence, due to the multifactorial genesis of neoplastic diseases, we cannot fully blame one factor - air pollution despite that how many substances formed it. Air pollution may not only increase the risk of incidence, but also worsen the prognosis of existing diseases. After consideration the above data, it seems reasonable to introduce screening tests for lung cancer - which would allow earlier and more beneficial treatment.

However, nothing will be able to replace the simplest method of fighting neoplastic diseases- education I We should increase awareness of risk factors and discourage smoking especially when each of us realizes that we passively smoke 0.4 package a day.

\section{References:}

1. WHO Air quality guidelines for particulate matter, ozone, nitrogen dioxide and sulfur dioxide - Global update 2005

2. Liu Y, Xie S, Yu Q, Huo X, Ming X, Wang J, Zhou Y, Peng Z, Zhang H, Cui X, Xiang H, Huang X, Zhou T, Chen W, Shi T. Short-term effects of ambient air pollution on pediatric outpatient visits for respiratory diseases in Yichang city, China. Environ Pollut. 2017 Aug;227:116-124.

3. Yue S, Wang Y, Wang J, Chen J. Relationships between lung cancer incidences and air pollutants. Technol Health Care. 2017 May 26.

4. https://sojp.wios.warszawa.pl/?page=c6h6

5. Kowalska M, Kocot K. Short-term exposure to ambient fine particulate matter (PM2,5 and PM10) and the risk of heart rhythm abnormalities and stroke

6. Pun VC, Kazemiparkouhi F, Manjourides J, Suh HH. Long-Term PM2.5 Exposures and Respiratory, Cancer and Cardiovascular Mortality in American Older Adults. Am J Epidemiol. 2017 May 24.

7. WHO Air quality guidelines for particulate matter, ozone, nitrogen dioxide and sulfur dioxide. World Health Organization 2006.

8. REGULATION OF THE MINISTER OF THE ENVIRONMENT of August 24, 2012 on the levels of certain substances in the air.

9. Database Main Inspectorate of Environmental Protection

10. Wojciechowska Urszula, Didkowska Joanna. Morbidity and mortality from malignant tumors in Poland. National Cancer Registry,-- The Maria Skłodowska-Curie. Institute Oncology Center. Available at http://onkologia.org.pl/raport... access from 04/09/2020

11. van der Zee SC, Fischer PH, Hoek G. Air pollution in perspective: Health risks of air pollution expressed in equivalent numbers of passively smoked cigarettes. Environ Res. 2016 Jul;148:475-483. doi: 10.1016/j.envres.2016.04.001. Epub 2016 Apr 30. PubMed PMID: 
27136673.

12. Directive 2008/50/EC of the European Parliament and of the Council of 21 May 2008 on ambient air quality and cleaner air for Europe. 